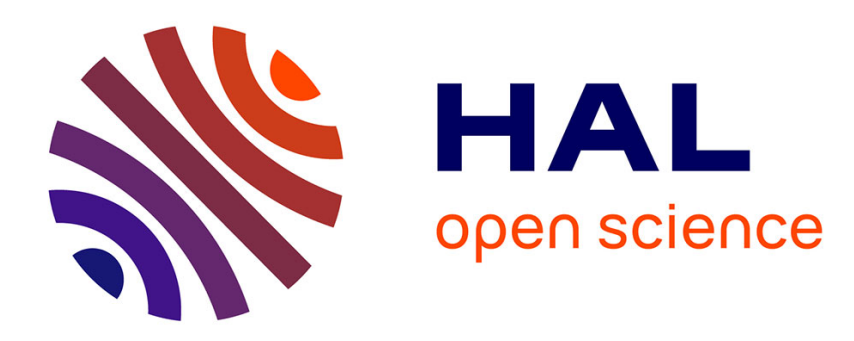

\title{
Sur l'absorption atmosphérique des radiations ultra-violettes
}

\author{
A. Cornu
}

\section{To cite this version:}

A. Cornu. Sur l'absorption atmosphérique des radiations ultra-violettes. J. Phys. Theor. Appl., 1881, 10 (1), pp.5-17. 10.1051/jphystap:01881001000500 . jpa-00237880

\section{HAL Id: jpa-00237880 https://hal.science/jpa-00237880}

Submitted on 1 Jan 1881

HAL is a multi-disciplinary open access archive for the deposit and dissemination of scientific research documents, whether they are published or not. The documents may come from teaching and research institutions in France or abroad, or from public or private research centers.
L'archive ouverte pluridisciplinaire HAL, est destinée au dépôt et à la diffusion de documents scientifiques de niveau recherche, publiés ou non, émanant des établissements d'enseignement et de recherche français ou étrangers, des laboratoires publics ou privés. 


\section{JOURNAL}

\section{DE PHYSIQUE}

\section{THÉORIQUE ET APPLIQUÉE.}

SUR L'ABSORPTION ATMOSPHÉRIQOE DES RADIATIONS OLTRA-VIOLETTES;

P.IR N. A. CORNL.

I. Sur la limite ultra-violette du spectre solaire. - Je me suis proposé, depuis plusieurs années, d'obtenir la limite extrême du spectre solaire du côté ultra-violet, c'est-à-dire du còté des courtes longueurs d'onde. La connaissance exacte de la limite du spectre ou plutôt de la loi suivant laquelle décroît l'intensité du spectre continu idéal de la photosphère apporterait, sur la difficile question de la température du Soleil, des données au moins aussi importantes que les mesures thermiques correspondant aur radiationà grande longueur d'onde.

Malheureusement, comme on va le roir, l'atmosphère exerce sur les radiations à courte longueur d'onde une absorption si énergique, que la majeure partic du spectre solaire ultra-ıiolet est. pour ainsi dire, brusquement interceptée d'une manière complète: l'étude que je m'étais proposée est donc actucllement impossible' sous la forme simple que j’avais imałnéce. Nínmoins, les résultals obtenus en rue de ces recherche's préscutent quelque intérèt. même au point de vue auquel je m’étais primitivement placé; ils jettent, en tout cas, un certain jour sur l'allure probable de l'absorption atmosphérique à l'autre evtrémité du spectre, moins facile à étudier, et montrent que le phénomène thermique est plus com- 
plexe que ne le suppose le mode expérimental généralement adopté pour l'évaluation de la température du Soleil.

Pour rester dans le domaine des faits et de leurs conséquences les plus immédiates, je me bornerai ici à donner un résumé succinct des expériences à l'aide desquelles j'ai cherché à obtenir photographiquement la limite ultra-violette du spectre solaire, l'analyse des conditions qui permettraient de reculer un peu cette limite et les vérifications expérimentales qui en dérivent.

Mode d'observation. - Le spectroscope dont je me sers généralement est composé d'un collimateur et d'une lunette, dont les objectifs sont respectivement formés par une lentille plan-convexe de quartz (d'environ $\mathrm{o}^{\mathrm{m}},{ }_{2} 6$ de longueur focale pour la raie $\mathrm{D}$ ); le prisme est en spath d'Islande : on utilise le ray on ordinaire, dont la dispersion est assez grande.

$\mathrm{Au}$ foyer de la lunette, on place une plaque fluorescente de verre d'urane pour la vérification approchée des réglages et finalement une petite glace sensibilisée par du collodion humide. La durée d'exposition varie, suivant les cas, de deux à six minutes, pour obtenir la limite extrême du spectre.

Au moyen d'un héliostat dont le miroir est remplacé par un prisme réflecteur en quartz, on concentre le faisceau de lumière sur la fente du collimateur, à l'aide d'une lentille de quartz.

La limite du spectre est variable suivant l'élat de l'atmosphère, la nature du collodion employé et la durée d'exposition; mais en choisissant les plus belles journées, en adoptant un collodion de composition constante et une durée d'exposition toujours la même, on obtient des séries très comparables ( ${ }^{1}$. Voici un exemple d'observation :

(') La composition du collodion et du révélateur que j'ai toujours employés de préférence est la suivante :

\begin{tabular}{|c|c|c|c|c|c|}
\hline $\begin{array}{l}\text { Collodion } \\
\text { (méler sans } \\
\text { précaution } \\
\text { et agiter). }\end{array}$ & $\begin{array}{l}\text { Pyroxyle } \ldots \ldots \ldots \\
\text { Alcool ............. } \\
\text { Éther............ } \\
\text { Iodure de cadmium. } \\
\text { Brom. de cadmium. }\end{array}$ & $\begin{array}{l}\mathrm{I}^{\mathrm{gr}} \\
40^{\mathrm{cc}} \\
60^{\mathrm{cc}} \\
1^{\mathrm{gr}} \\
\mathrm{o}^{\mathrm{gr}}, 25\end{array}$ & Révélateur. & $\begin{array}{l}\text { Eau distillée... } \\
\text { Sulfate de fer.. } \\
\text { Alcool......... } \\
\text { Acide acét. crist. }\end{array}$ & $\begin{array}{r}1000^{c c} \\
40^{g e} \\
30^{c c} \\
30^{c c}\end{array}$ \\
\hline
\end{tabular}

Le bain d'argent sensibilisateur contient de 7 à 8 pour roo d'azotate d'argent. Pour renforcer le cliché, on ajoute quelques gouttes d'un bain d'argent à 2 pour 100 au révélateur. 
1 I septembre 1878 . Observation faite a Courtenay 'Loiret'. Lutitude, $48^{\circ} \mathbf{2}^{\prime} \mathbf{2 0}^{\prime \prime}$; durée d'exposition, deux minutes et demie. Clichés renforcés une fois.

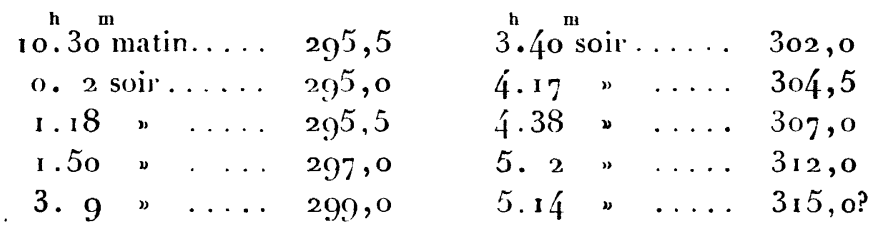

La limite du spectre est exprimée en longueurs d'onde, par comparaison avec la Carte que j'ai construite au mosen des observations faites pendant l'été de 1877 , et dont je reproduis ici la partie utile.

Fig. I.

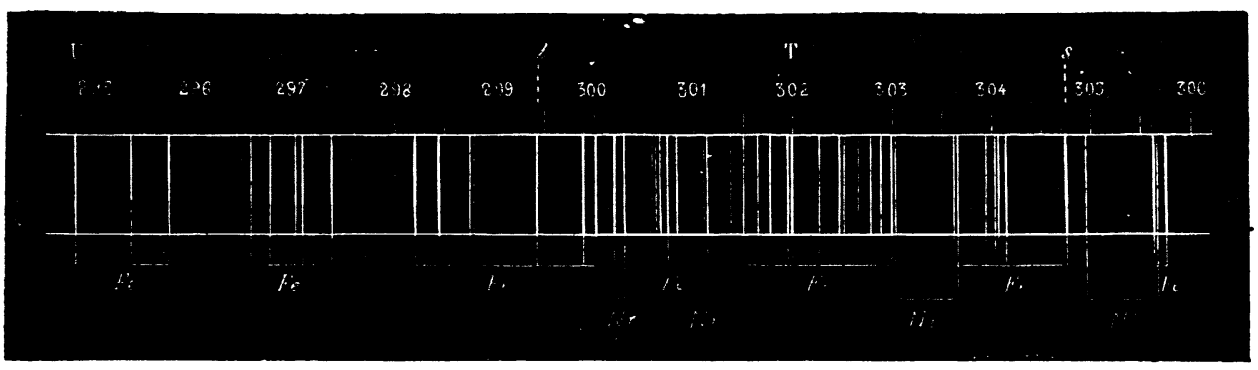

D’après le Tableau précédent, on voit que l'étendue du spectre diminue avec la hauteur du Soleil, ce qui tend à prouver que c'est à l'absorption de l'atmosphère qu'est due cette limitation.

Après divers essais faits d'après cette manière de voir, j'ai été conduit à représenter par la courbe suivante les résultats des observations. On porte comme abscisse la longueur d'onde limite observée et comme ordonnée le logarilhme du sinus de la hauteur. du Soleil (on sait que les épaisseurs atmosphériques traversées par les rayons solaires sont sensiblement en raison inverse du sinus de la hauteur du Soleil). L’ensemble des points ainsi définis se trouve pour une mème journée réparti sur une courbe à fort peu près rectiligne; les divergences proviennent d'altérations momentanées de la transparence de l'atmosphère. 
La construction des points correspondant à plus d'une centaine d'observations aux diverses heures de la journée m'a conduit à adopter comme ligne morenne la droite qui passe par le point $(\sin h=0,49$ et $\lambda=300)$ et parallèle à la direction définie par deux points $\left(\sin h=0,-5, \lambda=29^{5} ; \sin h=o, 30, \lambda=3 \circ 6\right)$ relevés sur une courbe dont les ordonnées représentaient les sinus des hauteurs vraies du Soleil au début de l'observation. On en déduit l'équation empirique $\sin h=0,49 \cdot 10^{-0,036173(\lambda-300)}$, ou, si l'on calcule avec des logarithmes népériens, $\sin h=0,49 e^{-0,08330(\lambda-300)}$.

La limite la plus éloignée que j'aie pu obtenir correspond à la longueur d'onde 293 ; elle a été atteinte asec certitude deux fois seulement, le 24 juin et le $\mathrm{s} 8$ août 1878 , aux environs de midi. Les radiations de $\lambda=294$ à 2.950 ont été atteintes bieñ des fois de mai à septembre 1877 et $\mathbf{1} 878$, et réccmment encore dès le 5 mai 1879 .

Malgré les nombreux essais faits dans les meilleures conditions, soit à Paris, soit à la campagne, il m'a été impossible d'aller plus loin.

Lorsqu'on examine les causes d'erreur qui peuvent modifier systématiquement les résultats, on reconnaît que la durée d'exposition des clichés est à peu près la seule qu'on ait à redouter dans l'appréciation de la limite du spectre; or il se présente une circonstance cxtrêmement favorable: c'est que l'effet photographique se produit pour ainsi dire dans les premiers instants, de sorte que la prolongation de l'exposition n'ajoute que peu à l'étendue des radiations agissantes. C'est ce quej'ai établi par une série méthodique d'expériences, parmi lesquelles je citerai la suivante comme typique :

16 octobre 1878 . Courtenay. $\mathrm{II}^{\mathrm{h}} 47^{\mathrm{m}}$, T. M.

Duréc de l'exposition Longueur d'onde (quatre épreuves sur le même cliché). limite du côté ultra-violet. Différences.

\begin{tabular}{|c|c|c|}
\hline $1^{s} \ldots$ & $\lambda=306,5$ & \\
\hline $5 \ldots \ldots \ldots$ & 3o r & 3,3 \\
\hline $20 \ldots \ldots \ldots$ & $29^{8,5}$ & $\begin{array}{l}2,0 \\
\mathbf{I}, 0\end{array}$ \\
\hline 100 & 297,5 & \\
\hline
\end{tabular}

Ainsi, les durées d'exposition variant à peu près en progression géométrique, les variations de la limite perceptible diminuent très 
rapidement; d’après la loi que suivent les différences, pour reculer la limite seulement d'une demi-unité, il faudrait plus que quadrupler la durée d'exposition et la porter à cinq ou six cents secondes ou dix minutes. Aussi l'e périence a-t-elle montré qu'il était à peu près indifférent de dépasser deux à trois minutes pour la durée de l'exposition lorsque le temps est bien pur; lorsque l'on craint le passage de brumes légères, il est plus prudent de prolonger cette durée jusqu'à six minutes, afin d'augmenter les chances d'éclaircie.

Analy se des conditions qui permettraient de reculer la limite. de l'observation. - La limitation du spectre ultra-violet paraissant due à l'absorption atmosphérique, peut-on espérer, en se plaçant dans des conditions plus favorables, reculer notablement la limite de visibilité? Nous allons voir, par l'analyse des conditions de l'erpérience, que, d'après les faits observés, on ne doit pas attendre une amélioration bien considérable ou du moins en rapport arec les difficultés matérielles qu'il faudrait affronter.

Exprimons l'intensité P de l'impression photographique en fonction des éléments qui la déterminent; nous admettrons que l'intensité $P$, pour des valeurs très petites (les seules sur lesquelles nous ayons ici à raisonner), est proportionnelle à l'intensité $\mathbf{J}_{\%}$ de la radiation de longueur d'onde $\lambda$ et à une fonction particulière de $\lambda$. et du temps $t ; \mathrm{P}=\mathrm{J}_{\lambda} \mathrm{F}(\lambda, \iota)$. Cette fonction $\mathrm{F}$ représente la sensibilité de la couche impressionnable. Si la radiation, avant d'agir, est transmise par un milicu d'épaisseur $\varepsilon$ as ant le pouvoir de transmission $a_{\lambda}$ pour la radiation $\lambda$, l'intensité devient $\mathrm{I}_{\lambda} ; \mathrm{I}_{i}=\mathrm{J}_{\lambda_{i}} a_{i}^{\varepsilon}, a_{i}<\mathrm{I}$. Le milieu absorbant étant limité par une surface sensiblement plane, la direction de transmission faisant un angle $h$ avec le plan, on a

$$
\varepsilon-\frac{l}{\sin h}
$$

$l$ est l'épaisseur normale du milieu abiorbant supporé homogìne; s'il n'est pas homogène, mais furmé de couches parallìles de densité variable $\delta, l$ représente la hauteur équivalente calculée par la formule $l \partial_{0}=\int \partial d z, \delta$ étant la densité correspondant à l'épaisseur $z$ et $\delta_{0}$ étant la densité choisie pour l'évaluation de la hauteur réduite l, car c'est la quantité de la matière traversée, et non 
sa répartition, qui produit l'absorption. Substituant dans la valeur de $\mathrm{P}$, on a

$$
\mathrm{P}=\mathrm{J}_{\lambda} \mathrm{F}(t, \lambda) a_{\lambda}^{\frac{l}{\sin /}}
$$

Il y a trois fonctions inconnues de $\lambda$, à savoir $\mathrm{J}, \mathrm{F}$ et $a_{\lambda}$; la forme particulière sous laquelle elles se présentent permet de les éliminer à l'aide de l'équation empirique fournie par les observations dont il a été question plus haut. En effet, on a déterminé pour un même lieu $\left(l=l_{1}\right)$ la loi qui lie les hauteurs $h$ du Soleil à la longueur d'onde limite $\lambda$ observée, c'est-à-dire à celle qui donne l'impression photographique limite $\mathrm{P}=w ; w$ est alors une constante, la même pour toutes les valeurs de $\lambda$. Prenant le logarithme des deux membres de l'équation précédente, où $\mathrm{P}=\mathfrak{w}, t=\mathrm{T}$, durée constante de l'exposition,

$$
\log w=\log \mathrm{J}_{\lambda}+\log \mathrm{F}(\mathrm{T}, \lambda)+\frac{1}{\sin h} \log a_{\lambda},
$$

qui se réduit à la forme $\frac{\sin h}{l}=\psi(w, T, \lambda)$, puisque $J_{\lambda}$ est une fonction de $\lambda\left(\mathrm{J}_{\lambda}\right.$ représente la loi qui lie l'intensité de la radiation avec la longueur d'onde dans le spectrc continu idéal de la photosphère).

Mais l'expérience a montré que dans le même lieu $\left(l=l_{1}\right)$, pour une durée de pose constante ( $\mathrm{T}=$ const.), la limite d'impression ( $\omega=$ const.) était donnée par la loi $\sin h=0,49 e^{-0,08330(\lambda-300)}$, ou de la forme

$$
\sin h=\mathbf{M} e^{-m\left(\lambda-\lambda_{0}\right)} .
$$

Substituant cette valeur de $\sin h$ et celle de $l$, la formule devient

$$
\frac{\sin h}{l}=\frac{M e^{-m\left(\lambda-\lambda_{n}\right)}}{l_{1}} .
$$

Variation de la limite avec l'altitude. - Si l'on se reporte à la formule primitive ( $\mathrm{I}$ ), on voit que l'absorption est d'autant moindre que la hauteur solaire est plus grande et que l'épaisseur $l$ est plus petite. On doit donc, en diminuant l'épaisseur atmosphérique, c'est-à-dire en augmentant l'altitude $z$ du lieu d'observation, étendre la limite de visibilité du spectre.

La formule (2) donne précisément la loi cherchée, c'est-à-dire 
ABSORPTION DES RADIATIONS CLTRA-VIULETTES. II

l'étendue gagnée avec l'altitude. En effet. la quantité $l$ est, à un facteur près, représentée par le poids d'une colonne atmosphérique ayant l'unité de base comme section: si donc on désigne par $p$ la pression barométrique, on aura $l=\mathbf{A} p$, où la loi de décroissance de la pression arec l'altitude est donnée par une expression de la forme $p=p_{0} e^{-\frac{\Sigma}{\Sigma_{0}}}, z_{0}$ étant la constante barométrique. Il vient finalement

$$
\sin h=\mathbf{N} e^{-m\left(\lambda-\lambda_{0}\right)-\left(\frac{z-z_{1}}{z_{0}}\right)},
$$

formule qui donne la loi approchée qui existe entre la longueur d'onde de la radiation du spectre solaire à la limite de visibilité pour une hauteur donnée $h$ du Soleil et l'altitude $z$ du lieu d'observation.

Nous pouvons maintenant savoir ce que l'on gagne par l'accroissement de l'altitude, à hauteur égale du Soleil; il suffit d'écrire que l'exposant de $e$ est constant.

La longueur d'onde de la radiation limite et l'altitude sont donc liées par une fonction linéaire, de sorte que leurs variations finies ou infiniment petites sont proportionnelles; le coefficient de proportionnalité est $-m z_{0}$,

$$
d z=-663^{m}, 3 d h
$$

en substituant, $z_{0}=79^{6} 63^{\mathrm{m}}$ (constante de Ramond, $18336^{\mathrm{m}}$, multipliée par le module des logarithmes vulgaires, ou o, 434294) et $m=$ o, o833.

Ainsi on a chance de reculer la limite de visibilité des radiations d'une quantité correspondant à une unité (millionième de millimètre) dans la longueur d'onde lorsqu'on s'élève de $663^{\mathrm{m}}, 3 \mathrm{cn}$ altitude.

Ce gain est, relativement à la longueur du spectre que donnent les étincelles d'induction ou l'arc électrique, extrêmement faible. Si l'on songe que l'on ne peut guère faire d'observations régulières au-dessus de $4000^{\mathrm{m}}$ d'altitude, on voit que le gain calculé n'atteint que six unités ou environ la moitié de la différence qui se présente de l'hiver à l'été; le résultat obtenu serait donc encore bien loin de correspondre aux efforts à dépenser pour aller installer des appareils à une altitude aussi grande. 
II. Observation de la limite ultra-violette du spectre solaire a diverses altitudes. - Je me suis proposé d'étudier par l'expérience directe la variation de cette limite, en installant mes appareils ordinaires d'observation en différents points des Alpes, à des altitudes convenablement choisies.

J'aurais désiré atteindre une altitude aussi grande que celle à laquelle plusieurs observateurs ont porté leurs appareils pour l'étude de la radiation calorifique du Soleil, par exemple au sommet du mont Blanc ou du Breithorn. Malheureusement, je reconnus bientôt que cela était presque impossible dans le cas de mes expériences : les manipulations photographiques exigent une installation spéciale, des abris contre le vent et la lumière, et les observations, pour être concluantes, demandent un temps beaucoup plus long que celui pendant lequel on peut rester dans ces hautes régions.

Devant ces difficultés, qu'on ne pourrait surmonter qu'avec de grandes dépenses, j'ai cru devoir me contenter pour cette fois d'altitudes modérées $\left(2600^{\mathrm{m}}\right)$, mais réunissant des conditions relativement confortables, afin de pouvoir, pendant toute la journée, effectuer à loisir une série complète d'observations. J'ai pensé que ce que l'on perdrait par défaut d'altitude serait largement compensé par le soin et la tranquillité d'esprit que l'observateur apporterait à ses opérations.

Mettant à profit les indications données par plusieurs savants habitués aux expériences dans les montagnes, je me suis installé d'abord au Riffelberg, dans le massif du mont Rose, à une altitude de $2570^{\mathrm{m}}$. Cette station, où se trouve un hôtel convenable, est l'une de celles où les probabilités de temps clair sont les plus grandes. J'ai été très farorisé sous ce rapport, car j'ai cu trois belles journées consécutives, les 24,25 et 26 juillet, pendant lesquelles j'ai obtenu vingt-neuf clichés du spectre solaire.

L'étude de l'influence de l'altitude étant en réalité une mesure différentielle, mesure à effectuer sur un élément très délicat et très fugace, il faut, pour arriver à une conclusion valable, non seulement que l'appareil d'obserration soit le même, mais encore que les circonstances de toute nature soient aussi identiques que possible. En conséquence, je tàchai de réaliser une véritable mesure différentielle en transportant mes appareils à quelques kilomètres du Riffelberg, mais à $2000^{\mathrm{m}}$ en contre-bas. Je m'installai dans le 
petit village de Viège, au confluent de la vallée de Zermatt et de la vallée du Rhòne (altitude, $657^{\mathrm{m}}$ ), et pendant la magnifique journée du 28 juillet 1879 je pus obtenir une série de quatorze clichés.

Dans ces conditions, la comparabilité des résultats est aussi complète que possible : mèmes appareils, mème installation improvisée, par suite mêmes erreurs sýstématiques, mêmes conditions atmosphériques. Aussi la différence de position de la limite ultraviolette est-elle très nette; on en verra plus loin la valeur numćrique.

Enfin, comme contre-épreuve, je répétai les observations au Rigi (àmoitié chemin entre le Rigi Staffel et le Rigi Kulm, à $16{ }^{\mathrm{m}}{ }^{\mathrm{d}} \mathrm{d}$ 'altitude). La journée du $\mathbf{1}^{\text {er }}$ aoùt fut très belle jusqu'à $2^{\mathrm{h}}$, puis des brumes légères survinrent, comme cela arrive fréquemment dans ces parages. J'obtins une série de dix clichés; la limite observée aux environs de midi est intermédiaire entre celles observées au Riffelberg et à Viège. Les jours suivants ne furent pas assez purs pour permettre de faire des observations utiles.

L'examen micrométrique des clichés a donné les résultats suivants pour les limites extrêmes du spectre solaire ultra-violet; ils sont exprimés en longueurs d'onde :

\begin{tabular}{|c|c|c|}
\hline & $\lambda$. & Altitude. \\
\hline Riffelberg. . . . . . . . . & $99^{3,2}$ & $2570^{m}$ \\
\hline Rigi. . . . . . . . . . . & 294,8 & I65o \\
\hline Viège........... & 295,4 & $66 o$ \\
\hline Diffërence (Riffel-Viège). & $-2,2$ & 1910 \\
\hline
\end{tabular}

Les nombres ci-dessus ćtant très comparables entre cus, on peut en tirer une valeur directe du coefficient d'accroissement de visibilité avec la hauteur, que j'avais déduit des observations faites à Paris ( $50^{\mathbf{m}}$ d'altitude) ou à Courtenay (Loiret) $\left(17^{\mathrm{m}^{\mathrm{m}}}\right.$ environ $)$. Ce coefficient, calculé d'après la formule empirique qui lie la limite ultra-violette à la hauteur du Soleil, a été trouvé plus haut égal à $663^{\mathrm{m}}$; les résultats ci-dessus montrent que ce coefficient est trop faible. En effet, la différence d'altitude de $1910^{\mathrm{m}}$ entre Viège et le Riffel n'a reculé la limite que de 2,2 unités, ce qui correspond à $868^{\mathrm{nm}}$ d'accroissement d'altitude par unité. 
La belle série du 25 juillet, faite au Riffelberg, permet d'obtenir une valeur très approchée de ce coefficient d'accroissement. L'ensemble des résultats est représenté par une expression de la forme

$$
\sin h=M e^{-m\left(\lambda-\lambda_{0}\right)},
$$

dans laquelle $m=0$, i 256 . On en conclut, suivant l'analyse exposée précédemment,

$$
d z=-m z_{0} d \lambda \text { ou } \quad d z=-896^{\mathrm{m}}, 3 d \lambda .
$$

Telle est la valeur théorique de ce coefficient, fondée sur des données précises. L'observation directe donne $868^{\mathrm{m}}$, en partant des observations du Riffel et de Viège : la concordance est donc aussi satisfaisante qu'on peut le souhaiter dans l'évaluation numérique de phénomènes aussi délicats et constitue une vérification des calculs théoriques qui lient les phénomènes observés en un seul point avec ceux qu'on observe à diverses altitudes.

III. Démonstration directe de l'absorption des radiations ultraviolettes par l'atmosphère. - La théorie précédente suppose que c'est l'atmosphère qui absorbe les radiations ultra-violettes suivant une progression extrêmement rapide lorsque la longueur d'onde diminue. J'ai réussi à montrer par une expérience directe cette absorption des radiations très réfrangibles.

Un tube de $4^{\mathrm{m}}$ de longueur, fermé à ses deux extrémités par deux lames de spath fluor, a été intercalé entre le collimateur et le prisme du spectroscope formé par des objectifs et des prismes de quartz.

Devant la fente du collimateur on fait jaillir une forte étincelle d'induction entre deux électrodes d'aluminium et l'on observe le spectre soit avec un oculaire fluorescent, soit avec un oculaire photographique : l'aluminium donne le spectre le plus étendu qu'on connaisse; les trois derniers groupes de raies sont les derniers termes de l'échelle conventionnelle bien connue (Journal de Physique, t. VIII, p. I87). Lorsque le tube est plein d'air, on ne voit aucune trace de la raie 32 du spectre de l'aluminium; mais, si l'on fait progressivement le vide, la raie 3 g gagne notablement en intensité, la raie 32 apparaìt bientôt et finalement surpasse en 
éclat la raie $3 \mathbf{\imath}$; la raie $3 o$, qui varie extrèmement peu, sert de repère et rend très faciles ces appréciations relatives d intensité. $\mathrm{Si}$ on laisse rentrer l'air dans le tube, les mêmes phénomènes se reproduisent en ordre inverse.

Ainsi, l'absorption des radiations très réfrangibles par l'atmosphère est démontrée : l'explication admise pour rendre compte de la variation de la limite ultra-violette du spectre solaire est donc légitime.

IV. Sur la loi de répartition suivant l'altitude de la substance absorbant dans l'atmosphère les radiations solaires ultra-violettes. - Les résultats précédents peuvent se résumer ainsi :

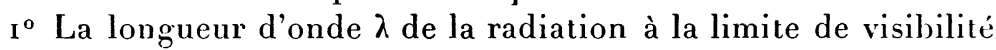
photographique observée dans le spectre ultra-violet, lorsque le Soleil est à une hauteur $h$ au-dessus de l'horizon, est donnée empiriquement par une expression de la forme

$$
\log \sin h=m \lambda+n
$$

$m$ et $n$ étant deux constantes; de sorte que, si l'on prend le logarithme sinus de la hauteur du Soleil comme ordonnée et la longueur d'onde comme abscisse, la ligne représentative des points ainsi définis, correspondant aux diverses heures de la journée, est une droite.

$2^{\circ}$ A des altitudes croissantes, la droite représentative se déplace parallèlement à elle-même, proportionnellement à la variation d'altitude, dans le sens d'un accroissement de visibilité du spectre; le taux de la progression $q$ est d'environ $868^{\mathrm{m}}, 2$, c'est-à-dire qu'à hauteur égale du Soleil on voit reculer d'une unité (millionième de millimètre) sur l'échelle des longueurs d'onde la limite visible lorsqu'on s'élève de $868^{\mathrm{m}}, \mathbf{2}$, de sorte que l'équation de la droite prend la forme

$$
\log \sin h=m\left(i+\frac{z}{q}\right)+n .
$$

La loi représentée par (3) ne peut ètre, à un point de vue rigoureux, qu'une loi approximative; on peut toutefois se demander quelles conséquences on pourrait en déduire si on la considérait comme exacte, sinon comme valeur numérique des coefficients, du 
moins comme forme mathématique; envisagée à ce point de vue, elle impose à la constitution optique de l'atmosphère des conditions qu'il est intéressant de mettre en évidence, car elles peuvent caractériser la nature des substances qui exercent l'absorplion et les lois qui régissent ce phénomène.

Pour traiter la question par le calcul, on ne peut pas, avec le nombre restreint des données expérimentales dont nous disposons, aborder le problème de la constitution de l'atmosphère dans toute sa généralité; nous allons nous borner au cas simple, et d'ailleurs très vraisemblable, dont voici l'énoncé :

On supposera que le pouvoir absorbant de l'atmosphère (formée de couches horizontales) est dì à une substance unique ou à un mélange homogène, entrant dans l'air pour une proportion variable el inconnue suivant l'altitude.

Le problème étant ainsi posé, les résultats précédents suffisent à déterminer la loi inconnue de répartition de la matière absorbante avec l'altitude et à exclure par là même certaines causes auxquelles on pouvait attribuer jusqu'ici l'absorption des radiations ultra$\checkmark$ iolettes.

Voici les résultats auxquels on parvient en traitant la question par le calcul ( $\left.{ }^{1}\right)$ :

La masse de la matière absorbante est à chaque altitude proportionnelle à la pression barométrique, par conséquent dans un rapport constant avec la masse de l'air atmosphérique.

Ce résultat exclut immédiatement la vapeur d'eau comme matjère absorbant les radiations ultra-riolettes. En effet, le poids de la vapeur d'eau est loin d'ètre en rapport constant avec le poids de l'atmosphère: la proportion diminue au contraire rapidement avec l'altitude.

On conclut des formules empiriques qui donnent le poids d'eau hygrométrique avec l'altitude que, si l'absorption des radiations ultra-violettes était due exclusivement ¿̀ l'action de la vapeur

(') Voir Comptes rendus des séances de l'Académie des Sciences, t. XC, séance du 26 avil 1880 . 
d'eau distribuée ayec l'altitude suivant la loi que l'expérlence indique, l'accroissement de visibilité daspectre solaire ultra-violet scrait d'une unité(millionième de millimètre) sur l'échelle des longueurs d'onde pour un accroissement d'altitude de $286^{\mathrm{n}} \cdot 9$. L'observation directe ayant donné trois fois plus, c'est-à-dire une unité pour $868^{\mathrm{m}}, 2$, il y a lieu de rejeter la vapeur d'eau comme cause exclusive de l'absorption des radiations ultra-riolettes.

Il est fort curieux que la vapeur d'eau, qui parait jouer le ròle prédominant dans l'absorption des radiations peu réfrangibles du spectre, ne soit pas la substance qui exerce l'influence principale dans l'absorption des radiations ultra-violettes.

J'ai tenu à vérifier ce résultat par une expérience directe : j’ai constaté que l'air saturé d'humidité à $\mathbf{1} \tilde{5}^{\circ}$ et l'air soigneusement desséché, observés sous une épaisseur de $4^{\mathrm{m}}$ dans l'appareil précédemment décrit (Comptes rendus, t. LXXXVIII, p. I29u), ne présentent aucune différence appréciable dans l'absorption qüils exercent sur les radiations très réfrangibles du spectre de l’aluminium.

L'eau, à l'état liquide, agit également d'une manière très différente sur les deux extrémités du spectre. M. Soret a montré, en effet, que l'eau distillée est parfaitement transparente pour les radiatións très réfrangibles (Archives de Geriève, mars 18;8), puisqu'une colonne d'eau distillée de $\mathrm{I}^{\text {in }}, \mathrm{I} 6$ laisse passer la radiation $n^{\circ} 28$ (spectre du zinc, $\lambda=2.06$ ); au contraire, pour les radiations infra-rouges, l'eau est si opaque, d'après les expériences de notre confrère M. Desains, que $o^{\text {m }}$, or d'épaisseur enlève au spectre calorifique des métaux incandescents la moitié de leur longueur et les trois quarts de leur intensité (Comptes rendus, t. LXYYJ. p. 286$)$. 